\title{
1 In vitro and in silico testing of partially and fully bioresorbable vascular scaffold
}

3 Authors:

4

5 Nenad Filipovic $^{1,2^{*}}$, Dalibor Nikolic ${ }^{1,2}$, Velibor Isailovic ${ }^{1,2}$, Miljan Milosevic ${ }^{1,2}$, Vladimir Geroski ${ }^{1,2}$, 6 Georgia Karanasiou $^{3}$, Martin Fawdry ${ }^{4}$, Aiden Flanagan ${ }^{4}$, Dimitris Fotiadis ${ }^{3}$ and Milos Kojic ${ }^{1}$

7

8 1) Bioengineering Research and Development Center, BioIRC, Kragujevac

9 2) Faculty of Engineering, University of Kragujevac

10 3) Foundation for Research and Technology Hellas, Greece

11 4) Boston Scientific Limited, Ireland

12

- Corresponding author: Nenad Filipovic, fica@kg.ac.rs, BioIRC Kragujevac, Faculty of Engineering, University of Kragujevac, Sestre Janjica 6, 34000 Kragujevac, SERBIA, phone: +38134334379. 
17

18

\section{Abstract}

Coronary artery disease (CAD), one of the leading causes of death globally, occurs due to the growth of atherosclerotic plaques in the coronary arteries, causing lesions which restrict the flow of blood to the myocardium. Percutaneous transluminal coronary angioplasty (PTCA), including balloon angioplasty and coronary stent deployment is a standard clinical invasive treatment for CAD. Coronary stents are delivered using a balloon catheter inserted across the lesion. The balloon is inflated to a nominal pressure, opening the occluded artery, deploying the stent and improving the flow of blood to the myocardium.

All stent manufacturers have to perform standard in vitro mechanical testing under different physiological conditions. In this study, partially and fully bioresorbable vascular scaffold (BVS) from Boston Scientific Limited have been examined in vitro and in silico for three different test methods: inflation, radial compression and crush resistance.

We formulated a material model for poly-L-lactic acid (PLLA) and implemented it into our in-house software tool. A comparison of the different experimental results is presented in the form of graphs showing displacement-force curves, diameter - load curves or diameter - pressure curves. There is a strong correlation between simulation and real experiments with a coefficient of determination $\left(R^{2}\right)>0.99$ and a correlation coefficient $(\mathrm{R})>0.99$.

This preliminary study has shown that in-silico tests can mimic the applicable ISO standards for mechanical in vitro stent testing, providing the opportunity to use data generated using in-silico testing to partially or fully replacing the mechanical testing required for regulatory submission.

Keywords: In vitro mechanical test, bioresorbable stent, PLLA, finite element analysis

Word count: 3388

Introduction: 569

Methodology for the mechanical simulations: 640

Results: 1728

Conclusions: 451 


\section{INTRODUCTION}

43 Coronary artery disease $(\mathrm{CAD})$ is one of the leading causes of death in the world. CAD occurs due to the

44 growth of atherosclerotic plaques in the coronary arteries. Percutaneous transluminal coronary angioplasty

45 (PTCA) was introduced as a minimally invasive treatment for CAD. The catheter balloon is inflated to a

46 nominal pressure, compressing the atherosclerotic plaque against the arterial wall and deploying the stent. This

47 restores the vessel patency and improves the flow of blood to the myocardium. PTCA generally results in high

48 clinical success rates, although a percentage of patients redevelop the initial symptoms over 6-12 months. This

49 re-narrowing of the treated artery is caused by restenosis, which is the principal limitation of this procedure

50 (Martin and Boyle, 2011).

51 Migliavacca et al. (2002) investigated the effect of the geometrical parameters (artery surface ratio, strut

52 thickness, cell length) of the Palmaz-Schatz stent on deployment characteristics. Results for this stent were 53 compared to the Carbostent and Multi-Link Tetra stent (Migliavacca et al., 2002). The expansion of each stent

54 was obtained through a pressure load applied to the inner surface. The results showed that geometrical

55 parameters had a significant influence on the deployment characteristics. When the metal to artery surface

56 ratio is lower, this is associated with higher rates of radial and longitudinal recoil and also lower rates of dog-

57 boning. This study demonstrated the potential of the finite element method in optimization of stent designs.

58 The design of biodegradable stents would be greatly improved if one were to predict how a given structure

59 degrades over time in response to cyclic loading. This coupled contact mechanics problem, while difficult

60 under typical physiologic conditions, is manageable with many FEM packages. In papers (Soares et al., 2007;

61 Soares et al., 2008; Soares et al., 2010a; Soares et al., 2010b) a description of material properties that include 62 the effects of mechanical deformation in conditions of accelerating biodegradation is proposed. This is a basic 63 model in which the material, at fixed degradation, is characterized with a neo-Hookean, purely elastic model.

64 The energy density function for neo-Hookean material is dependent on shear modulus and the first principal 65 invariant of the right Cauchy-Green deformation tensor. Shear modulus is typically a material constant that is 
66 estimated from load-deformation experiments, but in the case of biodegradable material, this material property

67 varies with time as material degrades. The parameter of degradation is 3D spatially and time dependent 68 degradation field, and for undegraded material it has value 0 , and for completely degraded material it has value 69 1. Soares et al. (2008) proposed a function for rate of deformation in the case of uniaxial extension.

70 As mentioned above, using biodegradable materials for implantable stents brings numerous advantages.

71 Unfortunately, their performance is not sufficiently well characterized, either in vivo or in silico.

72 In this study we initially created a finite element model for a partially BVS stent (SYNERGYTM BP 73 (Bioabsorbable Polymer) Everolimus-Eluting Platinum Chromium Coronary Stent - Boston Scientific 74 Limited) and then for a fully BVS stent (prototype polymeric bioresorbable stent made from PLLA - Boston 75 Scientific Limited). Then we introduced our specific material model based on experimental curves 76 implemented on the PLLA material model. The PLLA experimental curves were available referring to three 77 different strain rates: $0.001 \mathrm{~s}^{-1}, 0.01 \mathrm{~s}^{-1}$ and $0.1 \mathrm{~s}^{-1}$. In the Results section inflation, radial compression and 78 crush resistance tests are presented. A comparison of the results between simulation and real experiments is 79 given with coefficient of determination and correlation coefficient. 


\subsection{Finite element model for SYNERGYTM BP}

82

83

84

85

86

87

88

89

90

91

92

93

94

95

96

97

98

99

100

101

The SYNERGYTM BP Everolimus-Eluting Platinum Chromium Coronary Stent is a partially Bioresorbable Vascular Scaffold (BVS) produced by Boston Scientific Limited (Galway, Ireland). The geometrical model of the SYNERGYTM BP stent in pre-crimping configuration is presented in the Figure 1a. The strut section has $89 \mu \mathrm{m}$ width and $79 \mu \mathrm{m}$ thickness (Figure 1b). After crimping and expansion, the stent obtained has a nominal expanded inner diameter of $3 \mathrm{~mm}$ and a nominal length of $16.17 \mathrm{~mm}$.

\section{Figure 1}

Since the aim of the study is to perform structural analyses of the device, it is of primary importance to build a representative discretized model of the stent. The whole model was discretized with 36786 hexahedral elements (Figure 1c).

\subsection{Material model of SYNERGYTM BP stent}

The SYNERGYTM BP stent is made of a platinum-chromium alloy (Pt-Cr) which exhibits bi-linear elastoplastic behaviour.

For this device, the following material parameter values were used (O'Brien et al., 2010). The values for the material density, Young's modulus, Poisson's ratio and yield stress were provided by Boston Scientific Limited.

\subsection{Finite element model of a polymeric prototype of a bioresorbable stent implantation system}

The device is a BVS prototype polymeric bioresorbable stent made from PLLA, supplied by Boston Scientific Limited. Analogous to the SYNERGYTM BP case, the geometrical model of the polymeric prototype of a bioresorbable stent was provided by Boston Scientific Limited (Figure 2a). It has a length of $16 \mathrm{~mm}$, an 
102 103 104 105 106 107 108 109 110 internal diameter of $3 \mathrm{~mm}$, the same as the tube used for laser-cutting the stent, with $184 \mu \mathrm{m}$ strut width and strut thickness of $115 \mu \mathrm{m}$.

Figure 2

The whole model was discretized with 49464 hexahedral elements (Figure 2b).

This polymeric device is made of poly-L-lactic acid (PLLA), an elasto-visco-plastic polymer, which exhibits a non-trivial mechanical behaviour dependent on both the strain rate and the operating temperature.

\subsection{Material model of BVS material}

The model used in PAK solver (Kojic and Filipovic) is based on experimental curves provided by manufacturer (Boston Scientific Limited), Figure 3a.

Figure 3

The manufacturer provided the average results of uniaxial tensile tests performed on a number of dog-bone samples with a gauge length $\mathrm{L}=5 \mathrm{~mm}$, width $\mathrm{W}=2 \mathrm{~mm}$ and a thickness $\mathrm{t}=0.105 \mathrm{~mm}$. Tests were conducted at three different temperatures: $25^{\circ} \mathrm{C}, 37^{\circ} \mathrm{C}$ and $48^{\circ} \mathrm{C}$. For each temperature, three different curves were available referring to three different strain rates: $0.001 \mathrm{~s}^{-1}, 0.01 \mathrm{~s}^{-1}$ and $0.1 \mathrm{~s}^{-1}$. Results are in accordance with typical PLLA behavior (Figure 3b): at each temperature, the three curves show a common initial elastic response, a strain rate dependent yield point and plastic behavior ending with a strong hardening. At higher temperature or lower velocity, stress values decrease despite the increasing final strains.

\subsection{Representation of the 3D stress-strain state by uniaxial experimental curves}

The principle of equivalence of virtual work in 1D and 3D stress-strain conditions is implemented. The following equivalent stress $\bar{\sigma}$ and equivalent strain $\bar{e}$ are adopted (Kojic and Bathe, 2005):

$$
\bar{\sigma}=\left\{\frac{1}{2}\left[\left(\sigma_{11}-\sigma_{22}\right)^{2}+\left(\sigma_{22}-\sigma_{33}\right)^{2}+\left(\sigma_{33}-\sigma_{11}\right)^{2}+6\left(\sigma_{12}^{2}+\sigma_{23}^{2}+\sigma_{31}^{2}\right)\right]\right\}^{1 / 2}
$$


$123 \bar{e}=\left[\frac{2}{3}\left(e_{11}^{2}+e_{22}^{2}+e_{33}^{2}\right)+\frac{1}{3}\left(\gamma_{12}^{2}+\gamma_{23}^{2}+\gamma_{31}^{2}\right)\right]^{1 / 2}$

124 where $\sigma_{i j}$ stress components; $e_{i j}$ are strains, with $\gamma_{i j}$ being engineering strains. The uniaxial constitutive 125 relationships obtained experimentally are used with these equivalent stress and strain, evaluated at each 126 integration points within the finite element model.

\section{$127 \quad 2.6 \quad$ Interpolation of experimental curves}

128 Uniaxial stress-strain curves for different strain rates and two temperatures are schematically shown in Figure $1293 b$.

130 We interpolate the equivalent stress for the current strain, stain-rate and temperature as

$$
\sigma_{T 1}=\left(\sigma_{1}+\frac{\sigma-\sigma_{1}}{\sigma_{2}-\sigma_{1}}\right)_{T 1}, \quad \sigma_{T 2}=\left(\sigma_{1}+\frac{\sigma-\sigma_{1}}{\sigma_{2}-\sigma_{1}}\right)_{T 2}
$$

132 and then interpolate for temperature,

$$
\sigma_{T}=\sigma_{T 1}+\frac{T-T_{1}}{T_{2}-T_{1}}\left(\sigma_{T 2}-\sigma_{T 1}\right)
$$

134 135 136 where it is assumed that $\sigma_{1}<\sigma<\sigma_{2}, \quad \mathrm{~T}_{1}<T<T_{2}$; the curves 1 and 2 correspond to strain rates $\dot{e}_{1}<\dot{e}<\dot{e}_{2}$ and temperatures $T_{1}$ and $T_{2}$. In the case of $\dot{e}<\dot{e}_{\text {min }}$ we use

$$
\sigma_{T 1}=\frac{\dot{e}}{\dot{e}_{\min }} \sigma_{1} \quad \text { or } \quad \sigma_{T 2}=\frac{\dot{e}}{\dot{e}_{\text {min }}} \sigma_{2}
$$

137 In the case of $\dot{e}>\dot{e}_{\min }$ we use 


\section{RESULTS}

140

141

142

143

144

145

146

147

148

149

150

151

152

153

154

155

156

157

158

159

160

161

162

\subsection{Inflation test}

The purpose of this test is to determine the diameter required to inflate the balloon to the nominal recommended pressure, analyzing the outer diameter (measured in three positions: proximal, middle, distal) and length. The test provides results for recoil, foreshortening, dog boning. Foreshortening test determines the length to diameter relationship of the stent, the unconstrained length of a self-expanding stent and the wall thickness of a stent. Dog boning test evaluates the difference between the diameter of the implant and those of the proximal and distal ends of the balloon, when the implant is released under the maximum recommended inflation pressure. This test also provides information that might be useful for planning of clinical treatment. The results from the test are diameter/pressure curves. The testing occurs at $37^{\circ} \mathrm{C}$ temperature in $\mathrm{pH} 7.4$ phosphatebuffered saline. For the test environment, $\mathrm{pH}$ and temperature are selected to simulate a clinically relevant environment.

For the in vitro experiment, a computer-controlled Nexus 5000 with $99.96 \%$ accuracy syringe pump was used for inflation. A pressure sensor with $0.1 \%$ measurement error and a Keyence laser optical micrometer was used for the measurement of diameter (Figure 4a). The sample is fixed in a specially designed fixator. At the start of the inflation process, on every increase of $50 \mathrm{kPa}$ the pump temporarily stops and performs the stent outer diameter measurement with the laser micrometer, and then the process continues. Stent manufacturers use a variety of methods to determine the optimal rate of stent inflation. Making the compromise between the rate of inflation and internal stress in the stent material. The aim is to perform the surgical procedure as quickly as possible without compromising the integrity of the stent too much. Finding this limit is a very demanding process and very much depends on the experience of the manufacturer. To obtain consistently test results the inflation speed used is around $3 \mathrm{kPa} / \mathrm{s}$ which is much less than the manufacturer's suggestion to provide the balloon material more time to adapt to new pressure and avoid possible inflation speed impact on the balloon material deformation. 
164 For in silico simulations, the stent is positioned in the way that the central stent axis is collinear with $\mathrm{Z}$ axis. 165 Node movement at one end of the stent is fixed in $\mathrm{Z}$ direction. Lower inflation speed provides numerical 166 stability. The pressure is set on the internal surface of the balloon, which is placed inside the stent. The contact 167 boundary condition is set between the stent internal surfaces and the balloon outer surfaces. Boundary 168 conditions for mimicking the test are presented in the Error! Reference source not found. Figure $4 \mathrm{~b}$.

\subsubsection{Computed results}

The stress distribution from the simulation of the inflation test for the SYNERGYTM BP device and BVS

171 prototype device are presented in Figure 5a and Figure 5b, respectively. In the case of the SYNERGYTM BP 172 device, the inflation test starts from a crimped device form without any residual stress and strain (material is 173 Pt-Cr alloy so residual stress and strain it is negligible and therefore was disregarded). But in the case of the 174 BVS prototype device (material PLLA), to preserve residual stress and strain, the crimping process is simulated 175 first (Figure 5b, top), then the inflation simulation is performed.

Figure 5

177 Comparison of diameter-pressure curve results, between real experimental data and simulations for the 178 SYNERGYTM BP and BVS devices are presented in Figure 6a and Figure 6b, respectively. In the real inflation 179 test 14 SYNERGYTM BP units and 9 BVS prototype units were tested, but only two representative diameter180 pressure curves from actual tests are presented in Figure 6a and Figure 6b. However, the data from all 181 SYNERGYTM BP; $n=14$ curves, and BVS prototype; $n=9$ curves were used in the calculation of the $\mathrm{R}^{2}$ results 182 reported in Table 1. 
The simulation of the inflation test also generates results for several different tests (Table 1):

186

187

188

189
- Foreshortening

- Dog boning

- $\quad$ Stent-free Surface Area.

Stent-free Surface Area (SAR) is easily measured from the CAD model and ISO 25539 part 2 does not require this parameter to be measured on a real test article. The simulation cannot provide a result for the Profile/diameter test that can be compared with the data generated from bench testing of an actual stent. During manufacturing process, a test article can be generated with different dimensions within the acceptable range of the profile/diameter specification. . However, the test can provide information about stent opening and the simulation can be programmed to expand the stent to a pre-defined diameter.

\subsection{Radial compression test (radial force)}

The purpose of this test is to determine the load/deformation characteristics of the stent, while a circumferentially uniform radial load is applied. The stent is compressed using a uniform rate of compression, starting with a diameter equal to the maximum indicated vessel diameter. In this simulation, the stent is positioned in such a way that the central stent axis is collinear with $\mathrm{Z}$ axis. Node movement at one end of the stent is fixed in the $\mathrm{Z}$ direction. The radial force is set on the outer surface of the cylinder placed outside of the stent with the purpose of simulating the "Mylar" loop crimping device. The contact boundary condition is set between the stent outer surfaces and the cylinder internal surfaces. Boundary conditions for mimicking the test are presented in Figure 7a (right).

Figure 7

\subsubsection{Computed results}

The stress distribution from the simulation of crimping the SYNERGYTM BP device and BVS prototype device have been presented in Figure $7 \mathrm{~b}$ and Figure 7c, respectively. 
Comparison of the diameter-load curve results, between real experimental data and the simulation is presented 209 in the Figure 7d and Figure 7e. In the real compression test 5 SYNERGYTM BP and 4 BVS prototype device 210 units were tested. The data from all SYNERGYTM BP: $n=5$ curves and BVS prototype device $n=4$ were used 211 in the calculation of the $\mathrm{R}^{2}$ results reported in Table 1.

212 Comparison of the real experimental data for stent recoil and the results of the simulation required 213 simplification in order to allow interpretation to take place. A comparison of the mean values between the real 214 test and the simulation is presented in Table 1.

\subsection{Crush resistance / Crush test with two plates (force / distance)}

217 The purpose of this test is to determine the load required to cause clinically relevant buckling or a deflection 218 equivalent to a diameter reduction of at least $50 \%$. In this test method, it is also necessary to determine the 219 load/deformation characteristics of the stent while a uniform axial load is applied. The stent is compressed 220 between two plates and a load force applied at a uniform rate. In this simulation, the stent is positioned in such 221 a way that the central stent axis is collinear with $\mathrm{Z}$ axis, between the two plates (top and bottom). Node 222 movement at one end of the stent is fixed in the $\mathrm{Z}$ direction. All stent nodes located in the $\mathrm{XZ}$ coordinate plane 223 are bounded in the $\mathrm{Y}$ direction and all stent nodes located in the $\mathrm{YZ}$ coordinate plane are bounded in the $\mathrm{X}$ 224 direction. The axial force is set on the top plate. The bottom plate is fixed. The contact boundary condition is 225 set between the stent outer surfaces and the surfaces of the plates. Boundary conditions for mimicking the test 226 are presented in Figure 8a.

Figure 8

\section{$228 \quad 3.3 .1 \quad$ Computed results}

229 The stress distribution from the crush test of SYNERGYTM BP and BVS prototype device have been presented 230 in Figure 8b and Figure 8c, respectively. 
231 Comparison of the diameter-load curve results, between real experimental data and the simulation is presented 232 in Figure 9a for SYNERGYTM BP devices and in Figure 9b for BVS prototype devices. In the real crush test 2335 SYNERGYTM BP units and 3 BVS prototype units were tested. The data from all SYNERGYTM BP: $\mathrm{n}=5$ 234 curves and BVS prototype device $n=3$ were used in the calculation of the $\mathrm{R}^{2}$ results reported in Table 1.

Figure 9

236 By comparing diameter-pressure curves (Inflation test: Figure 6a, Figure 6b) and diameter-load curves (Radial 237 compression test: Figure 7d, Figure 7e; Crush test: Figure 9a, Figure 9b) created from the simulation with data 238 from real experiments, it can be concluded that the simulation mimics the real inflation test with very high 239 precision. The precision of simulation is quantified by the coefficient of determination $\left(\mathrm{R}^{2}\right)$ and correlation 240 coefficient $(\mathrm{R})$ calculated between the simulation curve and all points from all experimental curves.

241 - From the obtained inflation test results there is a strong correlation for SYNERGYTM BP $\left(\mathrm{R}^{2}=0.999\right.$, 242 $\mathrm{R}=0.9955)$ and $\mathrm{BVS}\left(\mathrm{R}^{2}=0.9973, \mathrm{R}=0.9925\right)$ for the proximal/distal stent positions. At the central position for the SYNERGYTM BP stent, the coefficient of determination $\left(\mathrm{R}^{2}=0.777\right)$ and correlation coefficient ( $\mathrm{R}=0.8815)$ show a little "deviation", because the speed of process transition is different between the real test and the simulated test.

- In the case of the radial compression test it can be observed that the coefficient of determination $\left(\mathrm{R}^{2}=0.8099\right)$ and correlation coefficient $(\mathrm{R}=0.9)$ are lower in the case of the SYNERGYTM BP device. The difference is due to the setup for the radial mechanical test for a very small stent diameter. In the "Mylar loop" device setup if the stent diameter is decreasing, the measurement "noise" increases. In the real test measurements, when the diameter is less than $2 \mathrm{~mm}$, the load value is decreased. This directly produces lower results in the coefficient of determination (R2) and the correlation coefficient (R).

- Results from crush test present a strong correlation between the simulation and real experiments for the SYNERGYTM BP device, quantified with $\mathrm{R}^{2}=0.9957$ and $\mathrm{R}=0.9979$ and for the BVS device quantified with $\mathrm{R}^{2}=0.9986$ and $\mathrm{R}=0.9993$. 
256 


\section{CONCLUSIONS}

In this study, we presented in vitro and in silico mechanical tests with two different types of stent devices SYNERGYTM BP representing a partially BVS device and a prototype bioresorbable stent from Boston Scientific Limited representing fully BVS devices. A comparison between the results of the real test and the simulation are presented in the form of comparable curves. We presented inflation, radial compression and crush resistance tests. Comparison of the tests results interpreted in form of graphs (diameter - load curves or pressure - diameter curves) for the partially BVS SYNERGYTM BP (device already approved for commercialization) and the fully BVS devices (polymeric prototype of a bioresorbable vascular scaffold) show very good correlation with results from real mechanical tests. A newly developed material model for simulation of PLLA or other similar polymer materials shows very satisfying performance and results. We proposed a material model for PLLA in our program PAK (Kojic and Filipovic) for experimental curves which used original experimental curves. This material model includes average results of uniaxial tensile tests performed on a number of dog-bone samples and conducted at three different temperatures. The experimental curves show an initial elastic response, a strain rate dependent yield point and plastic behavior ending with a strong hardening. Any kind of experimental curves available for the stent material can be used, which is a significant benefit in comparison with commercial software where that is not possible. For most of the results, there are strong correlations between simulation and real experiments for the coefficient of determination $\left(\mathrm{R}^{2}>0.99\right)$ and the correlation coefficient $(\mathrm{R}>0.99)$.

In the inflation test for the SYNERGYTM BP stent, the coefficient of determination $\left(\mathrm{R}^{2}=0.777\right)$ and the correlation coefficient $(\mathrm{R}=0.8815)$ show a little "deviation" at the central stent position. This can be attributed to the speed of process transition between the real test and the simulation. In the radial compression test, the coefficient of determination $\left(\mathrm{R}^{2}=0.8099\right)$ and the correlation coefficient $(\mathrm{R}=0.9)$ are lower in the case of the SYNERGYTM BP device due to the setup of the radial mechanical test for a small stent diameter. 
All simulations need several hours of preparation for boundary conditions, prescribing material models, 281 material properties and nonlinear contact. The execution of the simulation takes a few hours which is attributed 282 to the complexity of most of the tests. The residual stress and initial geometry for different tests derive directly 283 from the previous running tests like crimping and expanding.

284 Up to our knowledge there is no similar study for comparison of partially and fully BVS. In addition, a newly 285 developed material model for simulation of PLLA that uses experimental curves has shown a very good 286 agreement with experimental test results. It has been shown that in-silico tests can mimic most of the 287 appropriate ISO standards for mechanical in vitro stent devices testing. It opens a new avenue for in silico tests 288 which can partially or fully replace the real mechanical testing required for submission to a regulatory body.

Acknowledgment

290 This study was funded by the European Project H2020 InSilc [grant number 777119] and Serbian Ministry of 291 Education, Science, and Technological Development [451-03-68/2020-14/200107 (Faculty of Engineering, 292 University of Kragujevac)]. This article reflects only the author's view. The Commission is not responsible for any 293 use that may be made of the information it contains.

294 This study was funded by the grants from the Serbia III41007, ON174028 and EC HORIZON2020 777119 295 InSilc project.

\section{References}

297 Auricchio F., Di Loreto M., Sacco E., 2001. Finite element analysis of a stenotic artery revascularisation 298 through a stent insertion. Computer Methods in Biomechanics and Biomedical Engineering 4: 249-263, DOI: 10.1080/10255840108908007

Bathe K. J., Finite Element Procedures, Prentice-Hall, Englewood Cliffs, N. J., 1996. 
Bergstrom, J. S., Boyce M. C., 1998. Constitutive modeling of the large strain time-dependent behavior of elastomers. Journal of the Mechanical and Physics of Solids 46:931-954, DOI: 10.1016/S00225096(97)00075-6.

Balossino R., Gervaso F., Migliavacca F., Dubini G., 2008. Effects of different stent designs on local hemodynamics in stented arteries. Journal of Biomechanics 41(5):1053-1061, DOI: 10.1016/j.jbiomech.2007.12.005

Capelli C., Gervaso F., Petrini L., Dubini G., Migliavacca F., 2009. Assessment of tissue prolapse after balloon-expandable stenting: influence of stent cell geometry. Medical Engineering and Physics 31(4):441-447, DOI: 10.1016/j.medengphy.2008.11.002

Chua D., MacDonald B., Hashmi M., 2004a. Finite element simulation of slotted tube (stent) with the presence of plaque and artery by balloon expansion. Journal of Materials Processing Technology 155-156:1772-1779, DOI: 10.1016/j.jmatprotec.2004.04.396

Chua D., MacDonald B., Hashmi M. 2004b. Effects of varying slotted tube (stent) geometry on its expansion behaviour using finite element method. Journal of Materials Processing Technology 155-156:1764-1771, DOI: 10.1016/j.jmatprotec.2004.04.395

De Beule M., Mortier P., Carlier S.G., Verhegghe B., Van Impe R., Verdonck P., 2008. Realistic finite element-based stent design: the impact of balloon folding. Journal of Biomechanics 41(2):383389, DOI: 10.1016/j.jbiomech.2007.08.014

Djukic T., Saveljic I., Pelosi G., Parodi O., Filipovic N., 2019. Numerical simulation of stent deployment within patient-specific artery and its validation against clinical data. Computer Methods and Programs in Biomedicine 175: 121-127, DOI: 10.1016/j.cmpb.2019.04.005

Dumoulin C., Cochelin B., 2000. Mechanical behaviour modelling of balloon-expandable stents. Journal of Biomechanics 33:1461-1470. DOI:10.1016/S0021-9290(00)00098-1 
Etave F., Finet G., Boivin M., Boyer J.C., Rioufol G., Thollet G., 2001. Mechanical properties of coronary stents determined by using finite element analysis. Journal of Biomechanics 34(8):1065-1075, DOI: $10.1016 / \mathrm{s} 0021-9290(01) 00026-4$

Hayman, D., Bergerson C., Miller S., Moreno M., Moore J. E., 2014. The effect of static and dynamic 328 loading on degradation of PLLA stent fibers. Journal of Biomechanical Engineering 136:081006, DOI: $10.1115 / 1.4027614$

Jovicic G., Vukicevic A., Filipovic N., 2014. Computational Assessment of Stent Durability Using Fatigue to Fracture Approach. Journal of Medical Devices, 8(4): 041002, DOI: 10.1115/1.4027687

Karanasiou G.S. et al., In Silico analysis of stent deployment- effect of stent design, 2018 40th Annual International Conference of the IEEE Engineering in Medicine and Biology Society (EMBC), Honolulu, HI, 2018, pp. 4567-4570, DOI: 10.1109/EMBC.2018.8513205.

Kojic M., Bathe K. J., 2005. Inelastic Analysis of Solids and Structures, Springer, Berlin-Heidelberg-New York.

Kojic M., Filipovic N., Stojanovic B., Kojic N., 2008. Computer Modeling in Bioengineering - Theoretical Background, Examples and Software. John Wiley and Sons, 978-0-470-06035-3, England

Kojic M, Filipovic N et al, http://www.bioirc.ac.rs/index.php/software/5-pak (last visited: June 10, 2021) 
347 Krsmanovic D., Filipovic N., Koncar I., Petrovic D., Milasinovic D., Davidovic L., 2014. Computer modelling of maximal displacement forces in endoluminal thoracic aortic stent graft. Computer Methods in Biomechanics and Biomedical Engineering, 17:9, 1012-1020, DOI:10.1080/10255842.2012.735661 implantation alters coronary artery hemodynamics and wall shear stress during maximal vasodilation. Journal of Applied Physiology 93:6, 1939-1946, DOI: 10.1152/japplphysiol.00544.2002

LaDisa J., Olson L., Guler I., Hettrick D., Audi S., Kersten J., Warltier D., Pagel P., 2004. Stent design properties and deployment ratio influence indexes of wall shear stress: a three-dimensional computational fluid dynamics investigation within a normal artery. Journal of Applied Physiology 97:1, 424-430, DOI: 10.1152/japplphysiol.01329.2003

Lally C., Dolan F., Prendergast P.J., 2005. Cardiovascular stent design and vessel stresses: a finite element analysis. Journal of Biomechanics 38(8):1574-1581, DOI: 10.1016/j.jbiomech.2004.07.022

Mac Donald B. J. 2007. Practical stress analysis with finite elements. Glasnevin Publishing: Dublin.

Martin D., Boyle F. J., 2011. Computational structural modelling of coronary stent deployment: a review. Computer Methods in Biomechanics and Biomedical Engineering 14:4, 331-348, DOI: $10.1080 / 10255841003766845$

Migliavacca F., Petrini L., Colombo M., Auricchio F., Pietrabissa R., 2002. Mechanical behavior of coronary stents investigated through the finite element method. Journal of Biomechanics 35(6):803-811, DOI:10.1016/s0021-9290(02)00033-7 
Migliavacca F., Petrini L., Montanari V., Quagliana I., Auricchio F., Dubini G., 2005. A predictive study of the mechanical behaviour of coronary stents by computer modelling. Medical Engineering and Physics 27(1):13-18, DOI: 10.1016/j.medengphy.2004.08.012

O'Brien B.J., Stinson J.S., Larsen S.R., Eppihimer M.J., Carroll W.M., 2010. A platinum-chromium steel for cardiovascular stents. Biomaterials 31(14): 3755-3761, DOI: 10.1016/j.biomaterials.2010.01.146.

Petrini L., Migliavacca F., Auricchio F., Dubini G., 2004. Numerical investigation of the intravascular coronary stent flexibility. Journal of Biomechanics 37:495-501, DOI: 10.1016/j.jbiomech.2003.09.002

Pontrelli G., de Monte F. 2007. Modelling of mass convectiondiffusion in stent-based drug-delivery. Paper presented at: XXV Congresso Nazionale UIT sulla Trasmssione del Calore; 18-20 May, 2007; Trieste.

Soares, J. S., Moore J. E. Jr., Rajagopal K. R., 2007. Modeling of biological materials. Edited by F. Mollica, L. Preziosi, K. R. Rajagopal. Birkhauser Basel, pp. 125- 177.

Soares, J. S., Moore J. E. Jr., Rajagopal K. R., 2008. Constitutive framework for biodegradable polymers with applications to biodegradable stents. ASAIO Journal 54(3):295-301, DOI: 10.1097/MAT.0b013e31816ba55a

Soares, J. S., Moore J. E. Jr., Rajagopal K. R., 2010a. Modeling of deformation-accelerated breakdown of polylactic acid biodegradable stents. Journal of Medical Devices 4:041007, DOI: $10.1115 / 1.4002759$

Soares, J. S., Rajagopal K. R., Moore J. E. Jr., 2010b. Deformation-induced hydrolysis of a degradable polymeric cylindrical annulus. Biomechenics and Modeling in Mechanobiology 9:177-186, DOI: $10.1007 / \mathrm{s} 10237-009-0168-\mathrm{Z}$ 
Soares, J.S., Moore, J.E., 2015. Biomechanical Challenges to Polymeric Biodegradable Stents. Annals of Biomedical Engineering 44, 560-579 (2016), DOI: 10.1007/s10439-015-1477-2

Vieira, A. C., Vieira J. C., Ferra J. M., Magalhaes F. D., Guedes R. M., Marques A. T., 2011. Mechanical study of PLA-PCL fibers during in vitro degradation. Journal of the Mechanical Behavior of 395 Biomedical Materials 4:451-460, DOI: 10.1016/j.jmbbm.2010.12.006

Vieira, A. C., Guedes R. M., Tita V., 2014. Constitutive modeling of biodegradable polymers: hydrolytic degradation and time-dependent behavior. International Journal of Solids and Structures 51:11641174, DOI: 10.1016/j.ijsolstr.2013.12.010

Vukicevic A.M., Stepanovic N.M., Jovicic G.R., Apostolovic S.R., Filipovic N.D., 2014. Computer 400 methods for follow-up study of hemodynamic and disease progression in the stented coronary artery by fusing IVUS and X-ray angiography. Medical and Biological Engineering and Computing, 52(6): 539-556, DOI: 10.1007/s11517-014-1155-9

$403 \quad$ www.bostonscientific.com/ (last visited: June 10, 2021)

404 Zienkievich, O. C., 1983. The finite element method, third edition, Published by McGraw-Hill Book Co., 405 New York, ISBN 10:0070840725 / ISBN 13:9780070840720, (1983)

Zunino P., D’Angelo C., Petrini L., Vergara C., Capelli C., Migliavacca F., 2009. Numerical simulation of 407 drug eluting coronary stents: mechanics, fluid dynamics and drug release. Computer Methods in 408 Applied Mechanics and Engineering. 198:3633-3644, DOI: 10.1016/j.cma.2008.07.019 